\title{
Design of Waste Management System for Smart Cities
}

\author{
Sandeep Dhawan \\ IT Director
}

\begin{abstract}
The vision of a smart city cannot achieve without a proper management system of solid waste. Waste management is a challenge of the new age; a huge amount of our waste is dependent on our population. Garbage containers are placed in public places are brimful due to an increase in waste. We are designing a management system for solid waste in which we use microcontroller and sensors are check the level of garbage in containers and after achieving the high level it sends the signal through Wi-Fi protocol to waste management department. Waste management department gives the route of the garbage truck. The electrical power of the garbage container is given by the solar power system to saving the electricity. In this paper Smart Garbage Management in Cities using IoT, Microcontroller, Sensors and Wi-Fi process. This system can be easily implemented in our city level.
\end{abstract}

Keyword - RF module, IR Sensors, microcontroller, Wi-Fi protocol.

\section{INTRODUCTION}

In our system, the Smart dustbins are linked to the internet to get the data of the smart dustbins. The hasty growth in population, mains to more waste dumping. So, a proper waste management system is compulsory to avoid diseases. Devices which controlled from the internet called as Internet of Things. Internet and its applications become an essential part of today's human life.

Smart dustbins are attached to the microcontroller-based system. IR Sensors and RF modules are attached to the microcontroller. IR sensor detects the level of the garbage in dustbin and RF Transmitter sends the signal to the RF receiver at the Central System and central system define the route of garbage trucks. For the power supply of smart dustbins, we use solar power system.

Abhay Shankar Bharadwaj et al. explained that "Smart City" is one such execution meant at civilizing the life of human beings and a conceptual tactic with an architectural key as a smart city application.[1] Pallavi K N et al. discussed the description of IoT and fundamentals, testing and prototyping tool simulator and lastly the study of many literatures available on smart waste management system using IOT. [2] Gopal Kirshna Shyam et al. provide a waste management solution, based on providing intelligence to waste bins, using an IoT prototype with sensors. [3] Parkash et al. design IoT Based Waste Management system. In which dustbins are provided with cheap embedded system it helps in tracking the garbage bins with their unique ID. [4] Mohammad Aazam at el. propose a cloud-based smart garbage management system in which the waste bins are prepared with sensors, capable of alerting their waste level and update the status to the cloud.[5] G.Jenilasree at el. confer on Smart Trash Management in Cities with IoT, Microcontroller and wi-fi process. This survey includes many ideas that can be simply applied in smart trash management [6]. Hitesh Poddar at el. presented an Integrated Podium for Waste Supervision where smart bins are equipped with a network of sensors and they transmit real time data indicating the fill ratio of the bin.[7] Prof. B.S. Malapur at el. Explain the IoT technolog with management of trash and manage trip in cities is done, so that rate and time are reduced with improved path for trash collection. [8] Sam Aleyadeh at el. propose an IoT based architecture that targets two elements. First monitoring the waste bulk and content in a waste bin. Secondly dynamic scheduling and routing of waste collection vans.[9] Krishna Nirde at el. Proposed internet of things based smart waste management scheme which allows waste management authorities to nonstop monitor dust bins placed at various locations and take appropriate schedules to collect it directly and efficiently.[10] Aksan Surya Wijaya et al. presented the smart waste dust bin that can managed the waste in a smart city. System consists sensors to measure the mass of waste and the level of waste inside the dust bin.[11] Andre Castro Lundin et al. explores the employing sensor allowed solutions to improve on waste monitoring and collection of trash bins. Through a usercentered design approach,a low-cost monitoring system developed and tested in study [12] Dr. N. Susila et al. present set-up a traditional method of manually visiting the places of the bins and cleaning them overflow of waste in municipal leads to the creation of dirty atmosphere which turns into hazardous for lives. Therefor system is established to seen the level of waste collected is intimated via messages so as to remove the excess of the junk dust bins.[13]

\section{BLOCK DIAGRAM}

In our system there are two main parts first is smart dustbin show in Fig.1 and other is in garbage control unit office shown in.Fig.2

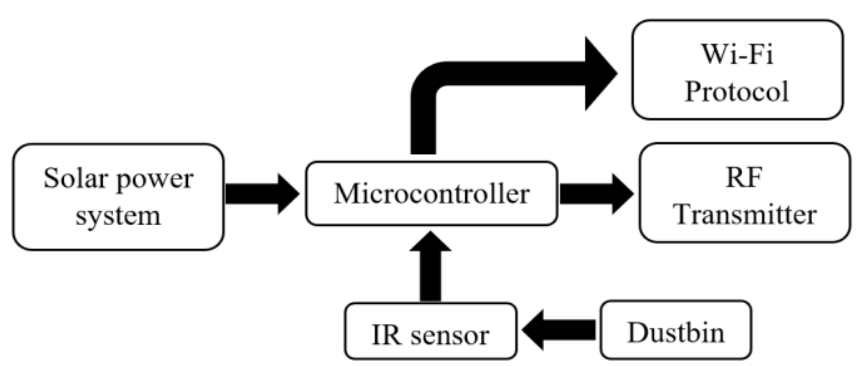

Fig. 1 Smart Dustbin 
In Fig.1 show the block diagram of smart dustbin system. In which the main power is providing by using the solar system. Each dustbin has its own power system. This power system is used to provide the power to the embedded system.IR Sensor checks the level of dustbins. when the level is high, the IR sensor gives the signal to the microcontroller and it sends a message through the RF transmitter to the garbage control unit. we are using as well as wi-fi system. If the distance between dustbin and office is high then we use wi-fi protocol. It's not compulsory to use both system at a time RF system and Wi-fi protocol.

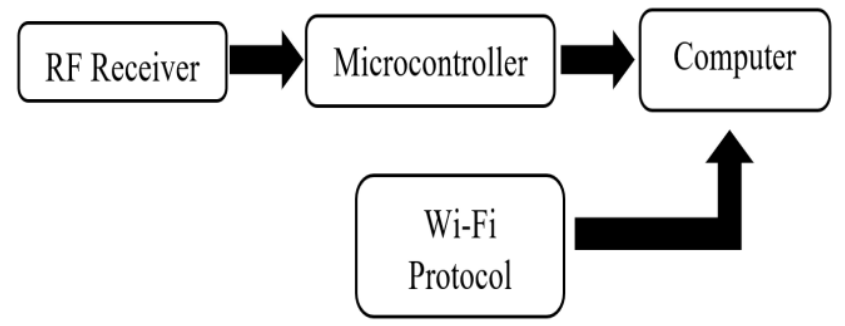

Fig. 2 Control Room

In Fig. 2 is the show in the office of the garbage control unit. In them, receive the signal from the RF Receiver and send it to the microcontroller, it's sent it to the computer for understandable.

After they receive all the information about bins control office will arrange a scheduled route of trucks. which can save time and money.

\section{METHODOLOGY}

For the battered environment we are built a smart dustbin in which we empty the bin before its overflow. In smart bins, we always check the level of garbage.

If garbage comes to a high level over system is send the signal to the garbage-controlled office and they will send garbage truck, which will empty the dustbin before its overflow. The flow chart of our system is shown in fig. 3

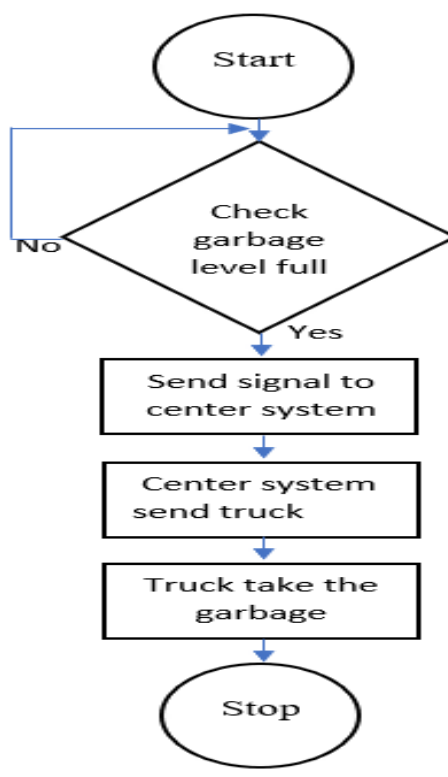

Fig. 3 Flow Chart

\section{DESIGN SYSTEM}

In our System, we use solar Power System, IR Sensor, Arduino microcontroller, RF Transmitter, RF Receiver, and the wi-fi protocol. Shown in fig. 4

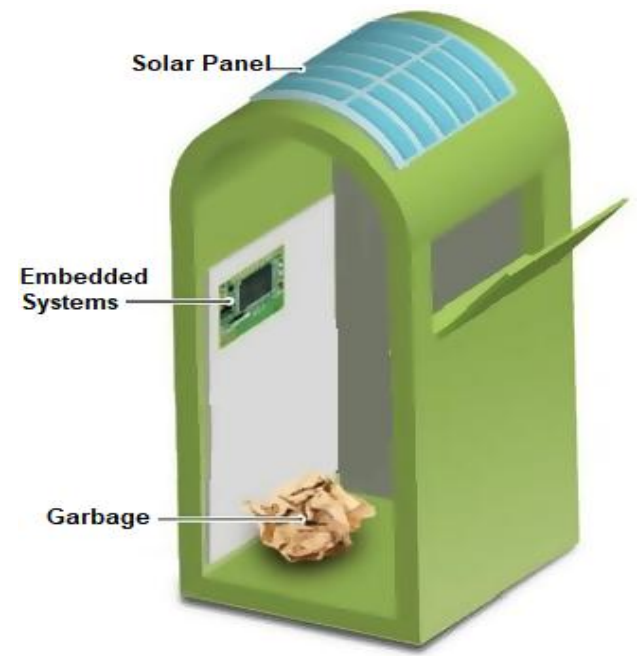

Fig. 4 Model of Smart Dustbin

In the Embedded system, we are installing the IR sensor, Arduino, RF Transmitter, and Wi-fi protocol.

The solar power system is used for the power of the whole system of the smart dustbin. IR sensor is used for the check the level of garbage. RF transmitter is used to send the signal if dustbin is full. Wi-Fi is used to link the bin with the control room or check the status of the bin.

\section{CIRCUIT DIAGRAM}

The circuit diagram of smart dustbin is shown in fig. 5 we are used in our circuit UNO Arduino, IR sensor and RF sensor. For the simulation of the circuit, we use Protus because it's easy to use and implementation. In this circuit, we are using serial communication port to check the massage of Arduino.

The solar system cannot show in the simulation of Protus because fix and stable voltage is available in Protus and if we simulate the solar system in protus then it will be a very complicated circuit and it's very difficult to understand the working of the circuit.

In our circuit we are used the UNO Arduino, IR sensor model FC51, 433Mhz RF Decoder Transmitter with Receiver, ESP8266 wifi module. These are elements are easily available in the market and easy to use and understand. 


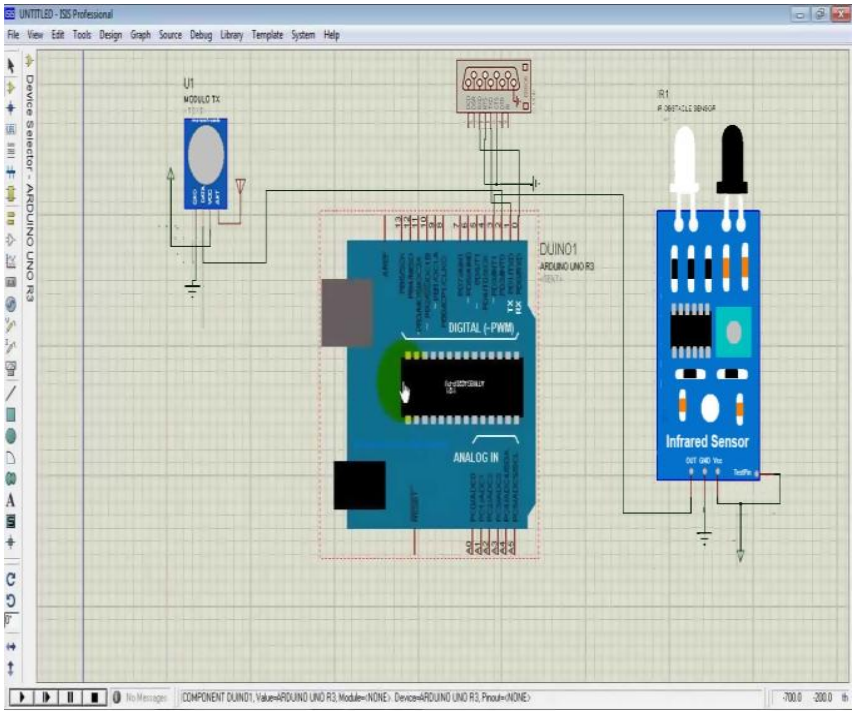

Fig. 5 circuit diagram

\section{WORKING}

The solar power system is used for the power of the whole embedded system of the smart dustbin.IR sensor FC-51 are used for the check the level of garbage. When garbage meets their highest level, UNO Arduino sends the signal through 433Mhz RF Decoder Transmitter, if dustbin is far away from control room then it sends a message through ESP8266 wi-fi module. the circuit of purpose system is shown in fig. 5

In the control room, all system is attached to the computer. They receive signal and shown on the computer.

When in control room person received the message, it sends the garbage truck which collects the garbage and avoids the unhygienic environment.

\section{CONCLUSION}

We are trying to provide hygiene and clean environment, implemented a real-time waste management system by using smart dustbins. It checks the fill level of dustbins whether the dustbin full or not. By applying this project, the fuel cost of truck reduction and actual usage of smart dustbins can be done. It's a portable system, low cost, and essay to implementation.

\section{FUTURE WORK}

The possibility for the future work of the system implemented very high. We can add the GSM System with our system. Add the phone number list with it. when the dustbin is full GSM system send the SMS to the relative truck driver. In this system, we save more time and more efficient system.

\section{REFERENCES}

[1] Abhay Shankar Bharadwaj, Rainer Rego, Anirban Chowdhury "IoT Based Solid Waste Management System" 978-1-50903646- 2/16/\$31.00 @2016 IEEE.

[2] Pallavi K N, Dr. Ravi Kumar V, Chaithra B M "Smart Waste Management using Internet of Things: A Survey" International conference on I-SMAC (IoT in Social, Mobile, Analytics and Cloud) (I-SMAC 2017).

[3] Gopal Kirshna Shyam, Sunilkumar S. Manvi, Priyanka Bharti "Smart Waste Management using Internet-of-Things" 2017 Second International Conference On Computing and Communications Technologies(ICCCT'17) 978-1-5090-62218/17/\$31.00_c 2017 IEEE.

[4] Parkash, Prabu V "IoT Based Waste Management for Smart City" International Journal of Innovative Research in Computer and Communication Engineering (An ISO 3297: 2007 Certified Organization)Vol. 4, Issue 2, February 2016.

[5] Mohammad Aazam, Marc St-Hilaire, Chung-Horng Lung, Ioannis Lambadaris "Cloud-based Smart Waste Management for Smart Cities" 978-1-5090-2558-9/16/\$31.00 @2016 IEEE

[6] G.Jenilasree, Dr.N.Shenbagavadivu, Dr.M.Bhuvaneswari "A Study on Automatic Solid Waste Management System for Smart City" International Journal of Creative Research Thoughts (IJCRT) Volume 5, Issue 4 November 2017 | ISSN: 2320-2882.

[7] Hitesh Poddar, Rituraj Paul, Sourangsu Mukherjee, Budhaditya Bhattacharyya "Design of Smart Bin for Smarter Cities" International Conference on Innovations in Power and Advanced Computing Technologies [i-PACT2017] 978-15090-5682-8/17/\$31.00 @2017 IEEE.

[8] Prof. B.S.Malapur, Vani R.Pattanshetti " IoT based Waste Management:An Application to Smart City" International Conference on Energy, Communication, Data Analytics and Soft Computing (ICECDS-2017).

[9] Sam Aleyadeh, Abd-Elhamid M. Taha "An IoT-based Architecture for Waste Management" 978-1-5386-43280/18/\$31.00 @2018 IEEE.

[10] Krishna Nirde, Prashant S. Mulay, Uttam M.Chaskar " IoT based solid waste management system for smart city" International Conference on Intelligent Computing and Control Systems ICICCS 2017.

[11] Aksan Surya Wijaya, Zahir Zainuddin, Muhammad Niswar "Design a Smart Waste Bin for Smart Waste Management" International Conference on Instrumentation, Control, and Automation (ICA) Yogyakarta, Indonesia, August 9-11, 2017 978-1-5386-0349-9/17/\$31.00 (02017 IEEE.

[12] Andre Castro Lundin, Ali Gurcan Ozkil "Smart cities: A case study in waste monitoring and management" Proceedings of the 50th Hawaii International Conference on System Sciences 2017 URI: http://hdl.handle.net/10125/41320 ISBN: 978-09981331-0-2 CC-BY-NC-ND

[13] Dr.N.Susila, Sruthi Anand, Dr.J.Granty Regina Elwin, Dr.T.Sujatha " Technology enabled Smart Waste Collection and Management system using IoT" International Journal of Pure and Applied Mathematics Volume 119 No. 12 2018, 1283-1295 ISSN: 1314-3395 (on-line version) url: http://www.ijpam.eu 